\title{
How Many Donor Colonies Should Be Cross-Fertilized for Nursery Farming of Sexually Propagated Corals?
}

\author{
Kenji Iwao $^{1}$, Naohisa Wada ${ }^{2}$, Aki Ohdera ${ }^{3}$, Makoto Omori ${ }^{1}$ \\ ${ }^{1}$ Akajima Marine Science Laboratory, Zamamison, Okinawa, Japan \\ ${ }^{2}$ Department of Marine Science and Resources, College of Bioresource Science, Nihon University, Kameino, \\ Fujisawa, Japan \\ ${ }^{3}$ Department of Biology, Eberly College of Science, The Pennsylvania State University, University Park, USA \\ Email: ${ }^{\text {iwao@amsl.or.jp }}$
}

Received 20 May 2014; revised 24 June 2014; accepted 2 July 2014

Copyright (C) 2014 by authors and Scientific Research Publishing Inc.

This work is licensed under the Creative Commons Attribution International License (CC BY).

http://creativecommons.org/licenses/by/4.0/

(c) (i) Open Access

\begin{abstract}
Coral reef restoration approaches have often utilized adult colonies as sources for fragments (i.e. clones) to be transplanted. Although restoration through this method is fast and cheap, it has been pointed out that it may reduce genetic diversity of the restored population. Low genetic diversity is a concern for reef restoration when seed fragments are raised asexually from only a few donor colonies. This can lead to lower fertilization rates among seed fragments, and reducing the longterm benefits of reef restoration in particular areas. Additionally, low genetic diversity can compound the effects of increased ocean temperature and other environmental stressors, further jeopardizing the health of a reef. An alternative approach through sexually propagated coral cultures and out-plantings can alleviate this problem. Sexually produced offsprings are more genetically diverse. They can be produced in far greater numbers than coral fragments and do not imply destructive methods. Ongoing research at the Akajima Marine Science Laboratory in Okinawa, Japan has helped to improve the production and maintenance of sexually propagated larval cultures. Our results show that crosses between gametes from 6 or more colonies will provide the highest fertilization rate (>95\%). Based on the results, we suggest the use of 6 or more donor colonies for practical gamete fertilization in sexually derived coral culture.
\end{abstract}

\section{Keywords}

Acropora, Coral Farming, Coral Reefs, Gamete Fertilization, Genetic Diversity, Reef Rehabilitation

\footnotetext{
${ }^{*}$ Corresponding author.
}

How to cite this paper: Iwao, K., Wada, N., Ohdera, A. and Omori, M. (2014) How Many Donor Colonies Should Be Cross-Fertilized for Nursery Farming of Sexually Propagated Corals? Natural Resources, 5, 521-526. 


\section{Introduction}

Currently, the most common approach to coral reef restoration is through the transplantation of fragments (i.e. clones). However, restoration practices that use a limited number of donor colonies can reduce gene flow [1]. Many broadcasting coral species do not self-fertilize [2] [3], and gametes from clone-mates are incapable of fertilizing each other. Furthermore, there is a significant negative relationship between fertilization rate and genetic similarity in branching corals [4]. It is ecologically important to maintain genetic/genotypic diversity within a coral population (see [5]). Low genetic diversity is a concern for reef restoration when seed fragments are raised asexually from only a few donor colonies. This can lead to lower fertilization rates among seed fragments, and reducing the long-term benefits of reef restoration in particular areas. Additionally, low genetic diversity can compound the effects of increased ocean temperature and other environmental stressors, further jeopardizing the health of a reef.

An alternative approach through the farming of coral offspring from larval cultures can alleviate these problems. The method involves the collection and cross-fertilization of gametes from gravid coral colonies during the spawning period, and the subsequent maintenance of the larval culture. Despite the potential cost, in addition to time that is required for this approach, it is a key solution that it could produce far greater numbers of offsprings than coral fragments and do not imply destructive methods which can impair donor coral population. Sexually produced offsprings are more genetically diverse, and provide long-term benefits than asexually produced coral fragments. As a result, higher fertilization rates can be expected from out-planted populations produced from sexually propagated offsprings than those from asexually raised fragments.

During past production of recruits using sexual propagation of acroporid corals, low fertilization rates were occasionally experienced when only a few donor colonies were used for the crosses. Although the reason for this is unknown, it was suspected that this occurred due to: 1) higher genetic similarity between donor colonies, 2) presence of morphologically indistinguishable cryptic species, or 3) the release of unhealthy gametes. Therefore, based on our experience, mixing the gametes from more than 3 donor colonies [6] or a minimum of 6 colonies [7] has been recommended to achieve higher fertilization rates (>95\%).

In the present study, fragments of gravid donor colonies from two locations in Kerama Islands, Okinawa, were collected and fertilization rates of crosses were compared by changing the number of colonies from 1 to 6 in order to corroborate our previous (above stated) recommendation experimentally.

\section{Methods}

In 2011, coral fragments (ca. 16 - 22 cm in length) from 13 gravid, donor colonies of Acropora tenuis were collected from the northern side of Yakabi Island $\left(26^{\circ} 13.070^{\prime} \mathrm{N}, 127^{\circ} 14.274^{\prime} \mathrm{E}\right)$ on June 5 and the northeastern side of Kuba Island $\left(26^{\circ} 10.711^{\prime} \mathrm{N}, 127^{\circ} 14.582^{\prime} \mathrm{E}\right)$ on June 11 (Figure 1). Distance between the two locations was approximately $5 \mathrm{~km}$. The donor colonies were distributed within a $150 \mathrm{~m}^{2}$ area at each location. Their fragments were collected using a hammer and chisel, and maintained in cages on the seafloor at approximately $2 \mathrm{~m}$ depth near Majanohama (Figure 1) in Aka Island before spawning. They were then brought to the laboratory in Aka Island on June 19 when bundles emerged at the mouth of the polyps and placed in separate $20 \mathrm{~L}$ buckets. Spawning occurred from 19:35 to 20:10 hr on the same night. The positively buoyant bundles were collected by gently skimming the water surface using a $100 \mathrm{~mL}$ polyethylene cup. The bundles were gently agitated in a small volume of filtered seawater to dissociate the egg and sperm. The eggs floating on the surface were collected with a pipette and rinsed twice with $10 \mu \mathrm{m}$ filtered seawater. The sperm suspensions were diluted to adjust their concentration. Three donor colonies (fragments) each from two locations were chosen for the cross-fertilization experiment. Egg and sperm collected from the 3 fragments from Kuba were designated A, B, C and a, b, c, respectively, while those from the 3 fragments from Yakabi Island were designated D, E, F, and d, e, f, respectively. Gametes were maintained in separate containers.

The gametes were then mixed in a pair-wise combination within glass vials, containing $50 \mathrm{~mL}$ seawater each. Sperm adjusted to a concentration of $10^{5} / \mathrm{mL}$ along with $300-470$ eggs were added to the vial for fertilization. The fertilization success can be influenced by competition between spermatozoids of distinct parents. Therefore, the concentration-adjusted sperm from 2 or more donor colonies was pooled together prior to mixing with eggs.

All combinations of crosses were conducted once within 3 hours after spawning. The gametes were allowed to fertilize for approximately 20 minutes in a room kept at approximately $25^{\circ} \mathrm{C}$, and percent fertilization was obtained by examining at least 100 eggs per cross under the dissecting microscope. Counts were obtained within 


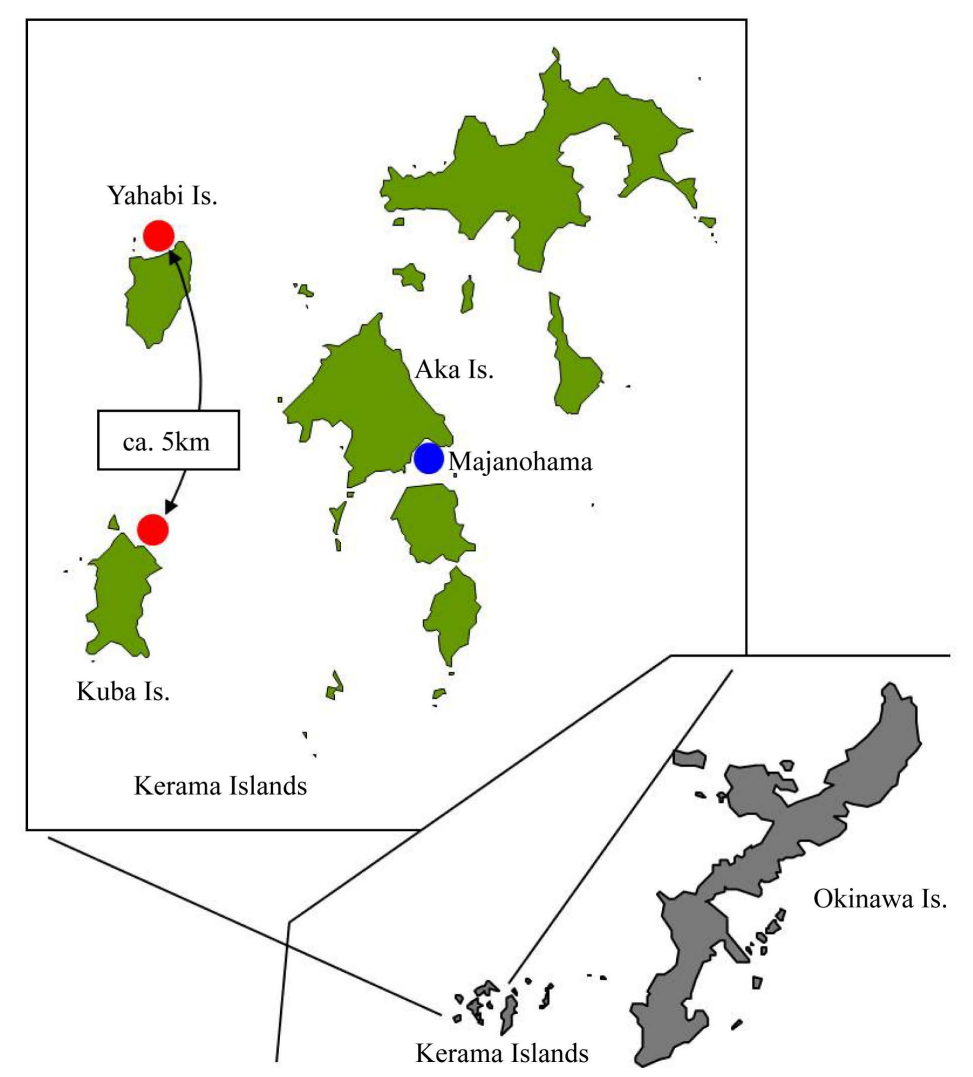

Figure 1. Map of Kerama Islands. Sampling locations of wild donor colonies (Kuba Island and Yakabi Island: red spots) and cultured colonies (Majanohama in Aka Island: blue spot).

5.5 hours of fertilization. In addition to the fertilization rate, the developmental stages of the embryos were scored. The presence of abnormally developing embryos, if any, was also recorded.

Cross-fertilization between 2 colonies were arranged as follows: $\mathrm{A} \times \mathrm{b}, \mathrm{A} \times \mathrm{c}, \mathrm{A} \times \mathrm{d}, \mathrm{B} \times \mathrm{c}, \mathrm{B} \times \mathrm{d}, \mathrm{B} \times \mathrm{e}, \mathrm{C} \times$ $\mathrm{d}, \mathrm{C} \times \mathrm{e}, \mathrm{C} \times \mathrm{f}$ (Figure 2(a)). The possibility of self-fertilization was also determined for comparison. The fertilization rate of 3-colony crosses between egg and sperm from Kuba Island (ABC $\times$ abc) and of 6-colony crosses between eggs from Kuba Island and sperm from Yakabi Island (ABC $\times$ def) were also compared. Finally, all gametes from the 6 colonies were mixed together (ABCDEF $\times$ abcdef).

Because of shortage of time, the experiment in 2012 was carried out using 6 colonies of $A$. tenuis from only Kuba Island (the same sampling location as 2011). The fragments were collected on June 1, and spawning occurred on June 11 (19:35 hr). The eggs (G, H, I, J, K, L) and sperm (g, h, i, j, k, l) from the 6 colonies were maintained in separate vials, and then mixed in a pair-wise combination following the procedure from the previous experiment. In addition to the six self-fertilization crosses, fertilization of eighteen 2-colony cross combinations, two 3-colony crosses (GHI $\times$ ghi and JKL $\times$ jkl), and three 6-colony crosses $(\mathrm{GHI} \times \mathrm{jkl}$, JKL $\times$ ghi and GHIJKL $\times$ ghijkl) were conducted (Figure 2(b)).

\section{Results}

Results of the cross-fertilization using 6 donor colonies in 2011 are shown in Figure 2(a). When gametes from 2 colonies were crossed, the fertilization rate varied considerably from $38.4 \%$ to $100 \%$. The fertilization rate was 77.2\% when the gametes of 3 colonies from Kuba Island ( $\mathrm{ABC} \times \mathrm{abc}$ ) were mixed, whereas, the cross between eggs of 3 colonies from Kuba Island and sperm of 3 colonies from Yakabi Island (ABC $\times$ def) resulted in much higher rate (98.2\%). It is possible that crosses between gametes from the 3 additional donor colonies, which were separated by greater geographical distance, provided additional genetic variation to the pool, thereby providing gametes with greater fertilization affinities. This was confirmed when gametes of all 6 colonies were 
(a)

\begin{tabular}{|c|c||c|c|c|c|c|c|}
\hline \multicolumn{2}{|c||}{} & \multicolumn{7}{c|}{ Eggs } \\
\cline { 2 - 9 } \multicolumn{2}{c|}{} & $\mathrm{A}$ & $\mathrm{B}$ & $\mathrm{C}$ & $\mathrm{D}$ & $\mathrm{E}$ & $\mathrm{F}$ \\
\hline \hline \multirow{4}{*}{$\approx$} & $\mathrm{a}$ & 0.8 & - & - & - & - & - \\
\cline { 2 - 9 } & $\mathrm{b}$ & 50.4 & 0.0 & - & - & - & - \\
\cline { 2 - 9 } & $\mathrm{c}$ & 38.4 & 96.4 & 0.0 & - & - & - \\
\cline { 2 - 9 } & $\mathrm{d}$ & 97.8 & 55.8 & 78.8 & 0.0 & - & - \\
\cline { 2 - 9 } & $\mathrm{e}$ & - & 100.0 & 100.0 & - & 0.0 & - \\
\cline { 2 - 9 } & $\mathrm{f}$ & - & - & 87.6 & - & - & 0.0 \\
\hline
\end{tabular}

(b)

\begin{tabular}{|c|c|c|c|c|c|c|c|}
\hline & & \multicolumn{6}{|c|}{ Eggs } \\
\hline & & G & $\mathrm{H}$ & I & $\mathbf{J}$ & K & $\mathrm{L}$ \\
\hline \multirow{6}{*}{$\begin{array}{l}\text { D. } \\
\text { के } \\
\text { के }\end{array}$} & $\mathrm{g}$ & 1.9 & - & - & 97.1 & 100.0 & 99.1 \\
\hline & $\mathrm{h}$ & 96.8 & 0.0 & - & - & 100.0 & 98.4 \\
\hline & i & 98.1 & 93.4 & 0.0 & - & - & 96.7 \\
\hline & $\mathrm{j}$ & 98.4 & 97.9 & 100.0 & 0.0 & - & - \\
\hline & $\mathrm{k}$ & - & 86.9 & 99.6 & 92.9 & 0.0 & - \\
\hline & 1 & - & - & 98.9 & 100.0 & 99.1 & 0.0 \\
\hline
\end{tabular}

Blue: 3 colonies $\mathrm{ABC} \times \mathrm{abc}$ : $77.2 \%$

Green: 6 colonies $\mathrm{ABC} \times \operatorname{def}: 98.2 \%$

Red frame: 6 colonies

ABCDEF×abcdef: $98.2 \%$
Blue: 3 colonies GHI $\times$ ghi: $97.2 \%$

Orange: 3 colonies JKL $\times \mathrm{jkl}: 96.8 \%$

Green: 6 colonies GHI $\times \mathrm{jkl}: 97.6 \%$

Pink: 6 colonies JKL $\times$ ghi: $100.0 \%$

Red frame: 6 colonies

GHIJKL×ghijkl: 99.5\%

Figure 2. Comparison of fertilization ratio (\%) between different combinations of 2-, 3-, 6-colony crosses. (a) Experiment in 2011; (b) In 2012. Number in each frame provides rates for 2-colony crosses. The rates for 3and 6-colony crosses are given in the margin.

mixed, which yielded high fertilization rate.

Instead of mixing gametes from donor colonies from 2 distinct sites in 2011, gametes from only Kuba Island were mixed in 2012. However, the fertilization rates between gametes from 2-, 3-, and 6-colony crosses were higher than those from the initial experiment (Figure 2(b)). All self-crosses resulted in $0 \%$ fertilization rate except the $\mathrm{G} \times \mathrm{g}$ cross which recorded a $1.9 \%$. Among fertilization rates between multiple colonies, the lowest percentage (86.9\%) was recorded in a 2-colony cross $(\mathrm{H} \times \mathrm{k})$. The 3 -colony crosses yielded a fertilization success of approximately $97 \%$, whereas all three 6 -colony crosses resulted in the rates exceeding $97 \%$.

In 2011 most of the embryos developed to or beyond the 128-cell stage between 5.5 and 7.0 hours after fertilization (Figure 3). However, five out of nine 2-colony crosses contained earlier stages, suggesting delayed embryogenesis. Additionally, two of 2-colony crosses contained abnormally developing embryos. Result of the experiments from 2012 was similar to that of 2011. Out of eighteen 2-colony crosses, nine contained developmentally delayed or abnormal embryos (data not shown).

\section{Discussion}

Genotypes of donor colonies used for the cross-fertilization were not determined in the present study. Use of colonies of unknown genotypes may reduce the potential for true crosses between distinct genotypes and may result in lower fertilization success. This may be the explanation for higher success in the 2012 experiment vs. the 2011 experiment. However, measurement of genetic similarity before fertilization is impractical for nursery farming of sexually propagated corals. The present cross-fertilizations were only conducted once for each combination, and therefore, were unable to confirm the results statistically. Lack of replicates may be criticized, but for the scope of this study, grasping general tendencies of the variation in fertilization rates was more meaningful than detailed analysis of the variation. It is important that the following similarities in outcome were obtained between the two years. 1) With 2-colony crosses, the fertilization rate varied widely; 2) with 3-colony crosses the fertilization rate increased but some showed relatively lower rates; and 3) with 6-colony crosses the rate was consistently higher than $95 \%$. 


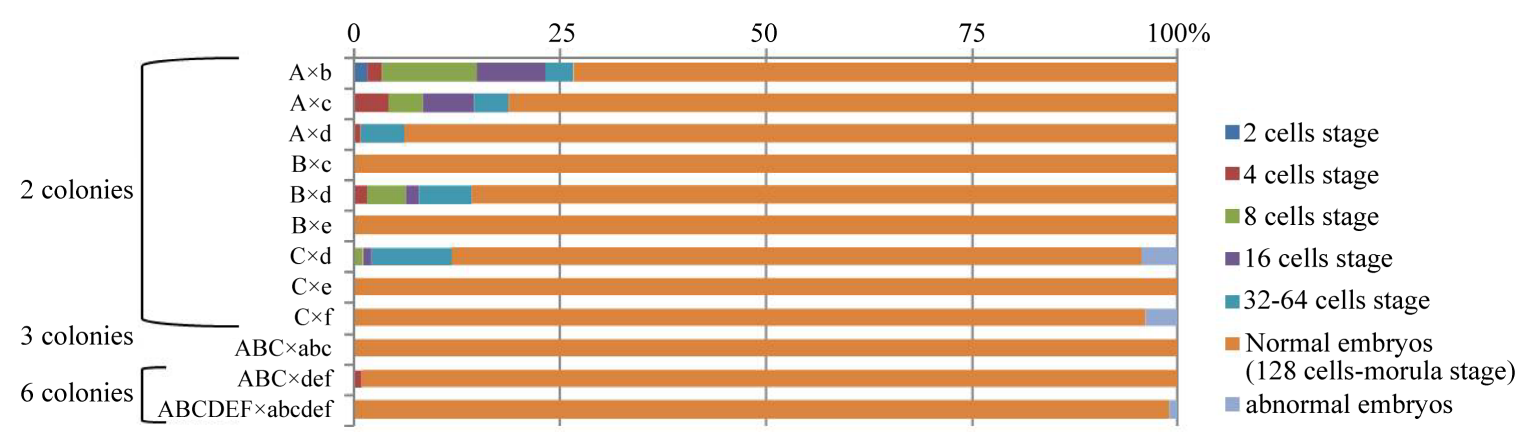

Figure 3. Composition of developmental stage of embryo originated from cross-fertilization between different donor colonies.

A rarefaction analysis of population genetic datasets conducted by Shearer et al. [8] showed that $>50 \%$ of the allelic diversity of a population could be retained by using gametes of 10 randomly sampled donor colonies, while 35 colonies would retain $90 \%$ of the original diversity. In practice however, it would be difficult and impractical to mix gametes from 35 gravid donor colonies to establish sexually propagated coral cultures. Since 2006, juvenile colonies of Acropora tenuis cultured after mixing gametes from 6 or more donor colonies were out-planted at Majanohama (Figure 1). They spawned initially at 4- and 5-year-old [9]. Spawning has continued every year since 2009. Fertilization rates among these 6 cultured colonies (5-year-old A. tenuis) were $>98.4 \%$ that was similar to those measured in crosses of 3 wild colonies [10].

In the present experiment, the number of donor colonies was limited to 6. It lacks replication and statistics, but shows evidence that fertilization rate increases with the number of donor colonies, as if there exists a threshold where the relationship reaches an asymptote. The purpose of the present study was not to assess how genotypic differences between the donor colonies affected fertilization rate, but aimed to investigate how many donor colonies are necessary to consistently achieve high fertilization rate. If the importance of allelic diversity of a population is taken into consideration, cross-fertilization with 10 colonies or more may be needed, but for practical nursery farming of sexually propagated corals, cross-fertilization with 6 or more colonies could be recommended in the laboratory.

\section{Acknowledgements}

We are grateful to H. Taniguchi who kindly collected coral colonies from Kuba and Yakabi Islands. Advices concerning laboratory experiments offered by M. Hatta and H. Fukami are also highly appreciated.

\section{References}

[1] Rinkevich, B. (2005) Conservation of Coral Reefs through Active Restoration Measures: Recent Approaches and Last Decade Progress. Environmental Science and Technology, 39, 4333-4342. http://dx.doi.org/10.1021/es0482583

[2] Heyward, A.J. and Babcock, R.C. (1986) Self- and Cross-Fertilization in Scleractinian Corals. Marine Biology, 90, 191-195. http://dx.doi.org/10.1007/BF00569127

[3] Willis, B., Babcock, R.C., Harrison, P.L. and Wallace, C.C. (1977) Experimental Hybridization and Breeding Incompatibilities within the Mating Systems of Mass Spawning Reef Corals. Coral Reefs, 16, S53-S65. http://dx.doi.org/10.1007/s003380050242

[4] Isomura, N., Baba, Y., Nagata, S., Nonaka, M. and Yamamoto, H.H. (2013) The Relationship between Genetic Similarity and Reproductive Success in the Branching Coral Acropora intermedia. Marine Biology Research, 9, 181-188. http://dx.doi.org/10.1080/17451000.2012.707321

[5] Baums, I.B. (2008) A Synopsis of Coral Restoration Genetics. In: Leewis, R.J. and Janse, M., Eds., Advances in Coral Husbandry in Public Aquariums. Public Aquarium Husbandry Series 2, Burgers’ Zoo, Arnhem, 335-338.

[6] Hatta, M., Iwao, K., Taniguchi, H. and Omori, M. (2004) Seed Production. In: Omori, M. and Fujiwara, S., Eds., Manual for Restoration and Remediation of Coral Reefs, Nature Conservation Bureau, Ministry of the Environment, Tokyo, 14-28.

[7] Guest, J.A., Heyward, A., Omori, M., Iwao, I., Morse, A. and Boch, C. (2010) Rearing Coral Larvae for Reef Rehabilitation. In: Edwards, A.J., Ed., Reef Rehabilitation Manual, Coral Reef Targeted Research \& Capacity Building for 
Management Program, St. Lucia, 73-92.

[8] Shearer, T.L., Porto, I. and Zubillaga, A.I. (2009) Restoration of Coral Populations in Light of Genetic Diversity Estimates. Coral Reefs, 28, 727-733. http://dx.doi.org/10.1007/s00338-009-0520-x

[9] Iwao, K., Omori, M., Taniguchi, H. and Tamura, M. (2010) Transplanted Acropora tenuis (Dana) Spawned First in Their Life 4 Years after Culture from Eggs. Galaxea, Journal of Coral Reef Studies, 12, 47. http://dx.doi.org/10.3755/galaxea.12.47

[10] Iwao, K. (2013) Reproduction of Outplanted Corals at Akajima Island. Midoriishi, 24, 24-25. (in Japanese) 
Scientific Research Publishing (SCIRP) is one of the largest Open Access journal publishers. It is currently publishing more than 200 open access, online, peer-reviewed journals covering a wide range of academic disciplines. SCIRP serves the worldwide academic communities and contributes to the progress and application of science with its publication.

Other selected journals from SCIRP are listed as below. Submit your manuscript to us via either submit@scirp.org or Online Submission Portal.
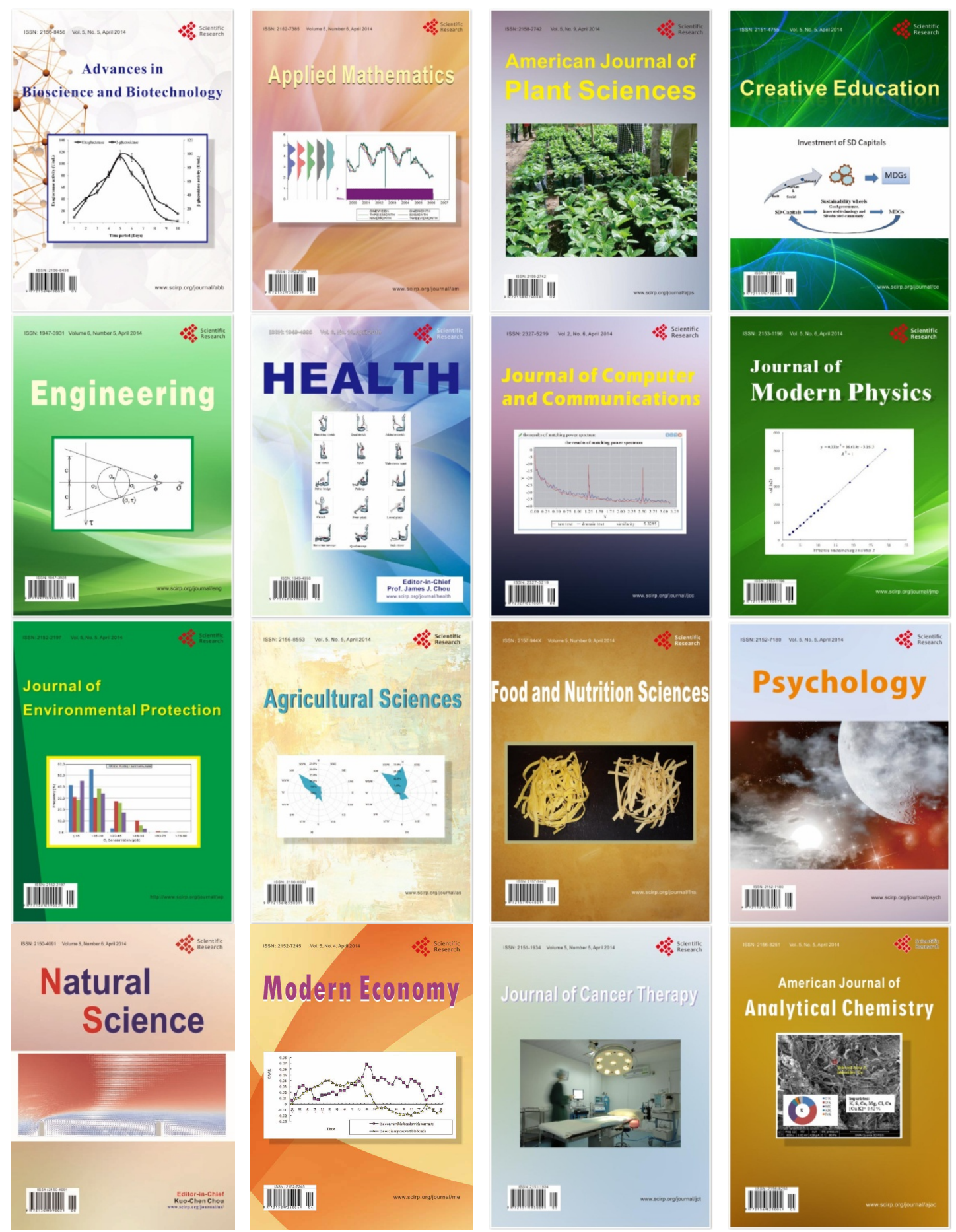